\title{
Transmuted Zeghdoudi Distribution: Theory and Applications
}

\author{
Molay Kumar Ruidas \\ Dept. Of Statistics, Trivenidevi Bhalotia College, Kazi Nazrul University, Raniganj, India \\ \& Research Scholar of West Bengal State University, Barasat \\ *Corresponding Author: molayruidas@gmail.com
}

Available online at: www.isroset.org

Received: 16/Mar/2020, Accepted: 13/Apr/2020, Online: 30/Apr/2020

\begin{abstract}
The Transmuted Zeghdoudi distribution(TZD) is derived using the Quadratic Rank Transmutation Map (QRTM) approach. Some mathematical properties of the proposed distribution are derived such as moments, skewness, kurtosis, mean residual lifetime, hazard rate, order statistics and reliability analysis etc. Estimation Properties like maximum likelihood estimation procedure is derived to obtain the estimates of parameters. A data set are analyzed to illustrate its applicability and to compare that this distribution performs better than many other distributions.
\end{abstract}

Keywords-Zeghdoudi Distribution, Quadratic Rank Transmutation Map, Moment Generating Function, Order Statistics, Maximum Likelihood Estimation

\section{Introduction}

Several Statistical distributions plays a very important role in prediction and analysis of real lifetime data. Data obtained from various fields such as financial, biological, physical, actuarial, engineering are fitted with the statistical distribution for prediction and analysis. Some well-known continuous distribution such as Exponential, Gamma, Lindley, Weibull, that have been applied to model the lifetime data sets. For the last 30 years, several new distributions were introduced that has a wide range of application in the field of data modelling. Due to the use of sophisticated technology, more and more data are obtained from various sources. So, research is going to develop new distribution that fits the data better and gives much more reliable estimates. Some of them one parameter mixture distribution such as Beta-Pareto[1], Lindley[2], BetaLaplace[3], Xgamma[4], Weibull- Pareto[5], Beta Exponentiated-Pareto[6],Transmuted Pranav Distribution[7], Transmuted Lindley Distribution[8], and Gamma-Lindley Distribution[9] and so on were used to fit the lifetime data better and have high flexibility. The Exponential families of distributions have played a vital role in probability distribution for modelling the real world phenomenon.

Recently, on parameter exponential families of distribution which is the mixture of $\operatorname{Gamma}(2, \theta)$ and $\operatorname{Gamma}(3, \theta)$ with mixing probability $\theta /(2+\theta)$ have been introduced and named as Zeghdoudi distribution[10]. The probability density function (pdf) of Zeghdoudi distribution is defined as

$$
g(x ; \theta)=\frac{\theta^{3} x(1+x)}{2+\theta} e^{-\theta x} \quad x>0, \theta>0
$$

and its cumulative distribution function (cdf) is given by

$$
G(x)=1-\frac{\theta^{2} x^{2}+\theta(\theta+2) x+\theta+2}{2+\theta} e^{-\theta x} \quad x>0, \theta>0 .
$$

We use the transmutation map approach which was suggested by Shaw et. al.[11]

$$
F(x)=(1+\lambda) G(x)-\lambda G^{2}(x), \quad|\lambda| \leq 1,
$$

where $G(x)$ is the cdf of the base distribution.

When $\lambda=0$ we have the distribution of the Zeghdoudi random variable. In this paper, we have introduced a transmuted version of the Zeghdoudi Distribution using Quadratic Rank Transmutation Map (QRTM) approach. The uses of this transmutation approach have played a vital role in modeling the lifetime data. We named the distribution as Transmuted Zeghdoudi Distribution (TZD).

The paper is organized as follows, Section II contains the introduction of pdf and cdf of Transmuted Zeghdoudi Distribution. Section III contains the mathematical properties of the distribution such as moments, moment generating function and second, third and fourth order moment. Section IV contains the measures of skewness and kurtosis and plotted for different values. Section V contains the order statistics. Section VI contains Reliability analysis and Hazard 
Function, Section VII contains simulation and parameter estimation using Maximum Likelihood Estimation., Section VIII contains the application to two real lifetime data and compare with two other distributions using related measures. Section IX concludes research work.

\section{Transmuted Zeghdoudi distribution}

Definition 4.1 A random variable $X$ is said to have the transmuted Zeghdoudi distribution with parameter $\theta$ and $\lambda$ if its probability density is defined as

$$
f(x)=\frac{\theta^{3} x(1+x) e^{-\theta x}}{2+\theta}\left[(1-\lambda)+2 \lambda\left(\frac{\theta^{2} x^{2}}{2+\theta}+\theta x+1\right) e^{-\theta x}\right] \quad ; \quad \theta, x>0,
$$
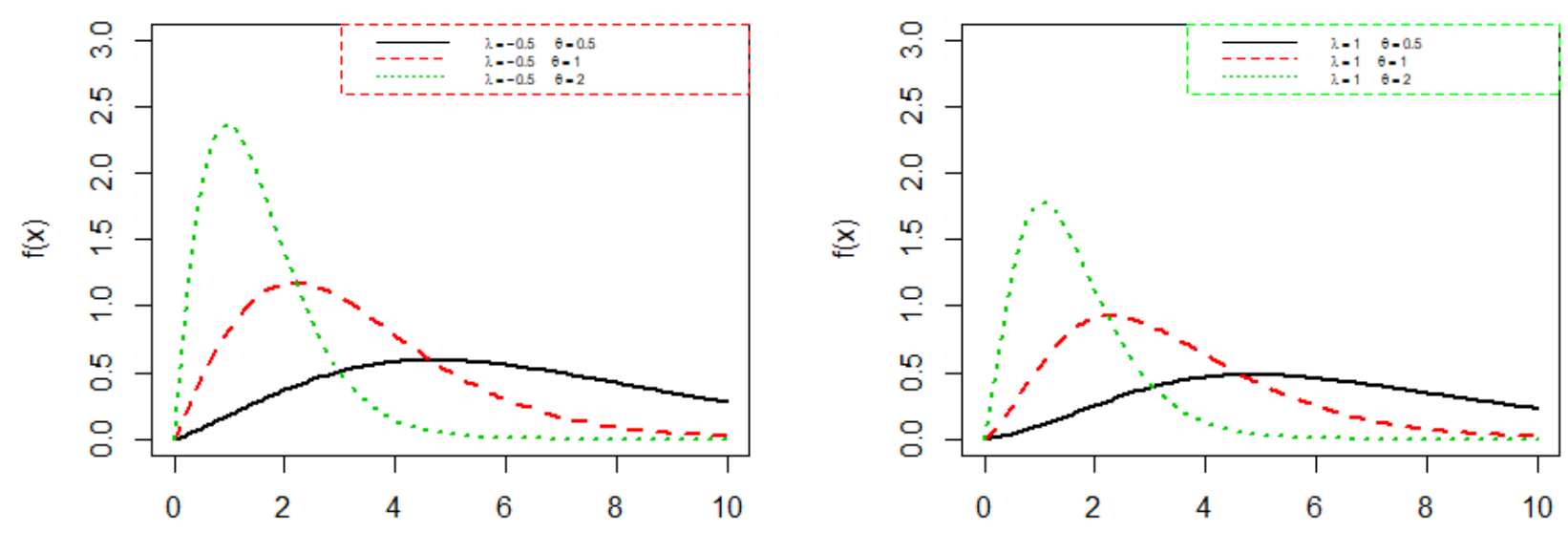

Figure 1: Plot of p.d.f of Transmuted Zeghdoudi Distribution

and its cdf is given by

$$
F(x)=\left[1-\left(\frac{\theta^{2} x^{2}}{2+\theta}+\theta x+1\right) e^{-\theta x}\right]\left[1+\lambda\left(\frac{\theta^{2} x^{2}}{2+\theta}+\theta x+1\right) e^{-\theta x}\right] \quad ; \quad \theta, x>0 .
$$
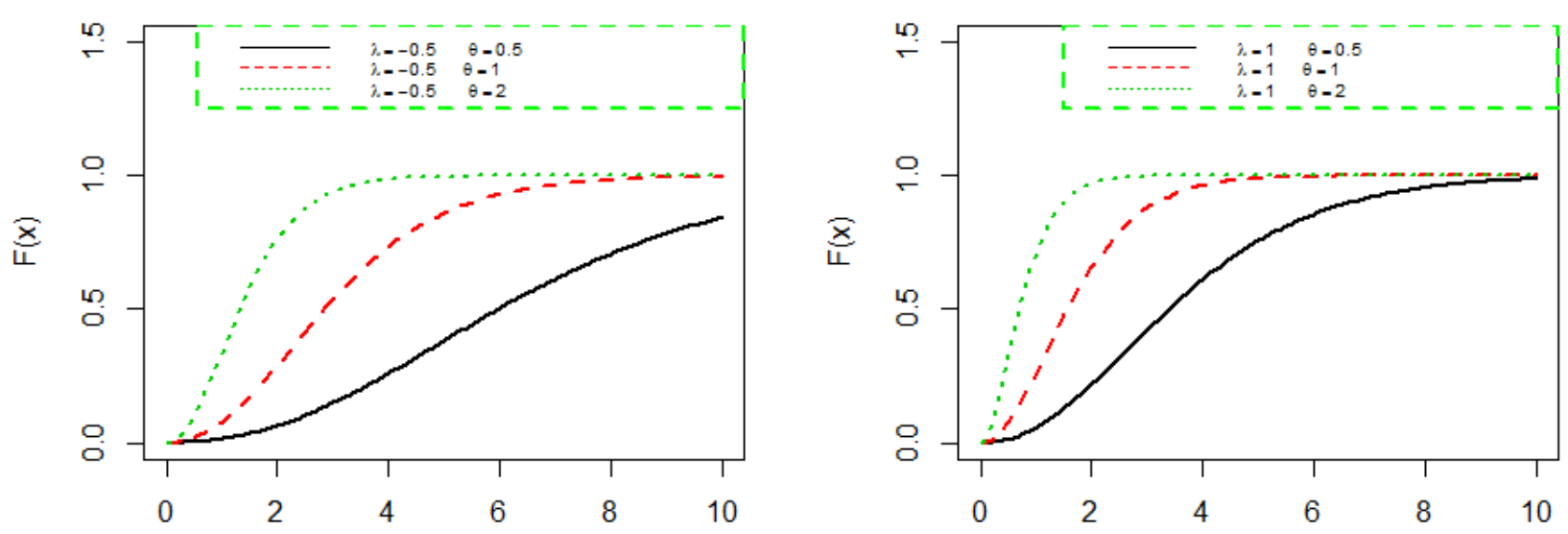

Figure 2: Plot of c.d.f. of Transmuted Zeghdoudi Distribution

The transmuted Zeghdoudi distribution is an extended model of Gamma distribution to analyse the more complex lifetime data.

\section{Moments and quantiles}

Let $\mathrm{x} \sim \mathrm{TG}(\theta, \lambda)$, then the $\mathrm{r}^{\text {th }}$ raw moment about the origin is given by

$$
E\left(X^{k}\right)=\int_{0}^{\infty} x^{k} \frac{\theta^{3} x(1+x) e^{-\theta x}}{2+\theta}\left[(1-\lambda)+2 \lambda\left(\frac{\theta^{2} x^{2}}{2+\theta}+\theta x+1\right) e^{-\theta x}\right] d x
$$


And the first, second, third and fourth order moment about origin are given by:

$$
\begin{aligned}
& \mu_{1}{ }^{\prime}=\frac{\theta^{3}}{2+\theta} \cdot\left[\frac{2}{\theta^{3}}\left(1+\frac{3}{\theta}\right)-\frac{2 \lambda}{\theta^{3}}\left(1+\frac{3}{\theta}\right)+\frac{48 \lambda \theta^{2}}{(\theta+2)(2 \theta)^{5}}\left(1+\frac{5}{2 \theta}\right)+\frac{12 \lambda \theta}{(2 \theta)^{4}}\left(1+\frac{2}{\theta}\right)+\frac{4 \lambda}{(2 \theta)^{3}}\left(1+\frac{3}{2 \theta}\right)\right], \\
& \mu_{2}{ }^{\prime}=\frac{\theta^{3}}{2+\theta} \cdot\left[\frac{6}{\theta^{4}}\left(1+\frac{4}{\theta}\right)-\frac{6 \lambda}{\theta^{4}}\left(1+\frac{4}{\theta}\right)+\frac{240 \lambda \theta^{2}}{(\theta+2)(2 \theta)^{6}}\left(1+\frac{3}{\theta}\right)+\frac{48 \lambda \theta}{(2 \theta)^{5}}\left(1+\frac{5}{2 \theta}\right)+\frac{12 \lambda}{(2 \theta)^{4}}\left(1+\frac{2}{\theta}\right)\right], \\
& \mu_{3}{ }^{\prime}=\frac{\theta^{3}}{2+\theta} \cdot\left[\frac{24}{\theta^{5}}\left(1+\frac{5}{\theta}\right)-\frac{24 \lambda}{\theta^{5}}\left(1+\frac{5}{\theta}\right)+\frac{1440 \lambda \theta^{2}}{(\theta+2)(2 \theta)^{7}}\left(1+\frac{7}{2 \theta}\right)+\frac{240 \lambda \theta}{(2 \theta)^{6}}\left(1+\frac{3}{\theta}\right)+\frac{48 \lambda}{(2 \theta)^{5}}\left(1+\frac{5}{2 \theta}\right)\right], \\
& \mu_{4}{ }^{\prime}=\frac{\theta^{3}}{2+\theta} \cdot\left[\frac{120}{\theta^{6}}\left(1+\frac{6}{\theta}\right)-\frac{120 \lambda}{\theta^{6}}\left(1+\frac{6}{\theta}\right)+\frac{10080 \lambda \theta^{2}}{(\theta+2)(2 \theta)^{8}}\left(1+\frac{4}{\theta}\right)+\frac{1400 \lambda \theta}{(2 \theta)^{7}}\left(1+\frac{7}{2 \theta}\right)+\frac{240 \lambda}{(2 \theta)^{6}}\left(1+\frac{3}{\theta}\right)\right] .
\end{aligned}
$$

As variance, skewness \& kurtosis calculative expressions are so complicate, therefore the graph of skewness \& kurtosis is plotted.
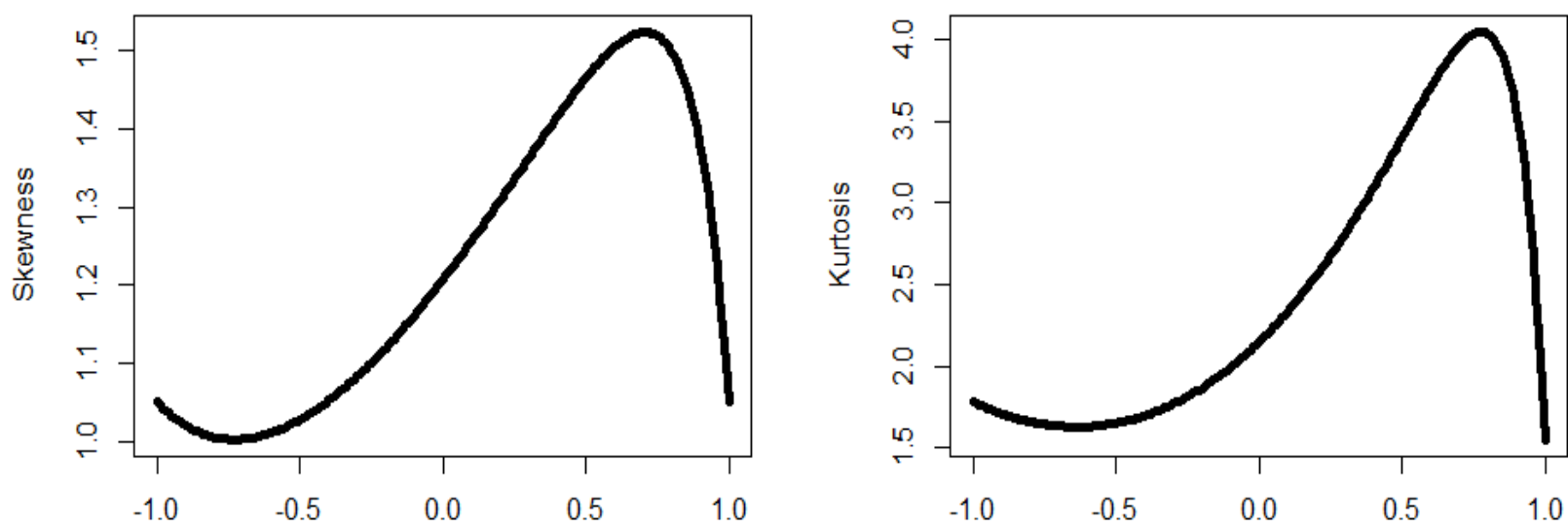

Figure 3: The graph of skewness and kurtosis for $\lambda$ from -1 to +1 .

\section{Moment Generating Function}

The moment generating function in terms of the moment are given by

$$
\begin{aligned}
M_{X}(t)=E\left(e^{t x}\right)= & \int_{0}^{\infty} e^{t x} \frac{\theta^{3} x(1+x) e^{-\theta x}}{2+\theta}\left[(1-\lambda)+2 \lambda\left(\frac{\theta^{2} x^{2}}{2+\theta}+\theta x+1\right) e^{-\theta x}\right] d x \\
= & \frac{\theta^{3}}{2+\theta}\left[\left(\frac{1-\lambda}{(\theta-t)^{2}}\right)\left(1+\frac{2}{\theta-t}\right)+\frac{12 \lambda \theta^{2}}{(2+\theta)(2 \theta-t)^{4}}\left(1+\frac{4}{2 \theta-t}\right)\right. \\
& \left.+\frac{4 \lambda \theta}{(2 \theta-t)^{3}}\left(1+\frac{3}{2 \theta-t}\right)+\frac{2 \lambda}{(2 \theta-t)^{2}}\left(1+\frac{2}{(2 \theta-t)^{3}}\right)\right]
\end{aligned}
$$

\section{Order Statistics}

Let $Y_{(1)}, Y_{(2)}, \ldots, Y_{(n)}$ be the ordered random sample drawn from the Transmuted Zeghdoudi Distribution, then the c.d.f. and p.d.f. of the $r^{\text {th }}$ order statistics is given by

$$
\begin{gathered}
G_{r}(y)=\sum_{i=r}^{n} n_{i}[G(y ; \theta ; \lambda)]^{i}[1-G(y ; \theta ; \lambda)]^{n-i}, \\
g_{r}(y)=\frac{n !}{(r-1) !(n-r) !}[G(y ; \theta ; \lambda)]^{r-1}[1-G(y ; \theta ; \lambda)]^{n-r} g(y ; \theta ; \lambda) .
\end{gathered}
$$

So, the p.d.f. and c.d.f. of $y_{(1)}$ and $y_{(n)}$ is given by

$$
\begin{gathered}
g_{y_{1}}(y)=n\left[\frac{\theta^{3} x(1+x) e^{-\theta x}}{2+\theta}\left((1-\lambda)+2 \lambda\left(\frac{\theta^{2} x^{2}}{2+\theta}+\theta x+1\right) e^{-\theta x}\right)\right]\left[1-\left(\frac{\theta^{2} x^{2}}{\theta+2}+\theta x+1\right) e^{-\theta x}\right]\left[1+\lambda\left(\frac{\theta^{2} x^{2}}{\theta+2}+\theta x+\right.\right. \\
\left.1) e^{-\theta x}\right]^{n-1}
\end{gathered}
$$




$$
\begin{gathered}
g_{y_{n}}(y)=n\left[\frac{\theta^{3} x(1+x) e^{-\theta x}}{2+\theta}\left((1-\lambda)+2 \lambda\left(\frac{\theta^{2} x^{2}}{2+\theta}+\theta x+1\right) e^{-\theta x}\right)\right]\left[1-\left(\frac{\theta^{2} x^{2}}{\theta+2}+\theta x+1\right) e^{-\theta x}\right]\left[1+\lambda\left(\frac{\theta^{2} x^{2}}{\theta+2}+\theta x+\right.\right. \\
\left.1) e^{-\theta x}\right]^{n-1}, \\
G_{y_{1}}(y)=1-\left(\left[1-\left(\frac{\theta^{2} x^{2}}{2+\theta}+\theta x+1\right) e^{-\theta x}\right]\left[1+\lambda\left(\frac{\theta^{2} x^{2}}{2+\theta}+\theta x+1\right) e^{-\theta x}\right]\right)^{n-1}, \\
G_{y_{n}}(y)=\left(\left[1-\left(\frac{\theta^{2} x^{2}}{2+\theta}+\theta x+1\right) e^{-\theta x}\right]\left[1+\lambda\left(\frac{\theta^{2} x^{2}}{2+\theta}+\theta x+1\right) e^{-\theta x}\right]\right)^{n} .
\end{gathered}
$$

\section{Survival Properties}

The hazard rate function or failure rate function for a continuous distribution with pdf $f(x)$, cdf $F(x)$ and survival function (sf) $\mathrm{S}(\mathrm{x})$ is defined as,

For the transmuted zeghdoudi distribution, the hazard rate function is given by

$$
h(x)=\ln \lim _{\Delta x \rightarrow 0} \frac{P(X<x+\Delta x \mid X>x)}{\Delta x}=\frac{f(x)}{1-F(x)}=\frac{f(x)}{S(x)} .
$$

$$
h(x)=\frac{\frac{\theta^{3} x(1+x) e^{-\theta x}}{2+\theta}\left[(1-\lambda)+2 \lambda\left(\frac{\theta^{2} x^{2}}{2+\theta}+\theta x+1\right) e^{-\theta x}\right]}{1-\left[1-\left(\frac{\theta^{2} x^{2}}{2+\theta}+\theta x+1\right) e^{-\theta x}\right]\left[1+\lambda\left(\frac{\theta^{2} x^{2}}{2+\theta}+\theta x+1\right) e^{-\theta x}\right]}
$$
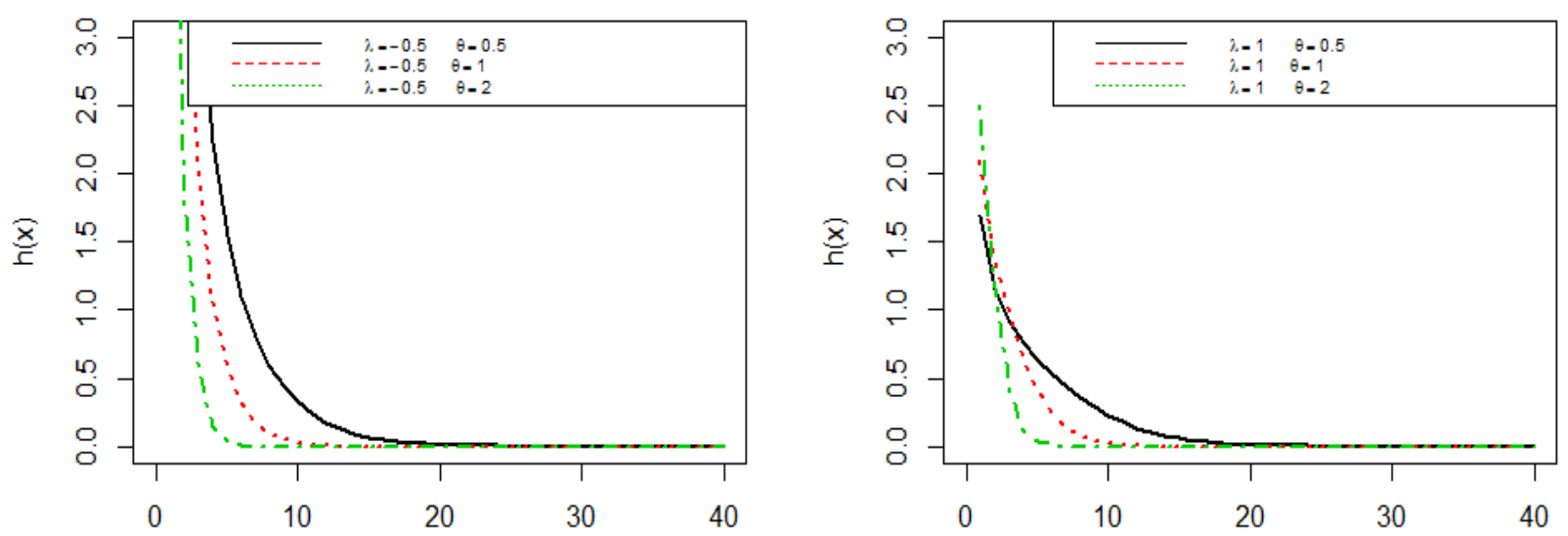

Figure 4: Plot of hazard function of Transmuted zeghdoudi distribution.

\section{Estimation of the parameters}

follows

Let $X_{1}, X_{2}, \ldots, X_{n}$ be a random sample of size $\mathrm{n}$ drawn from the pdf in (4). The log likelihood equation is as

$$
L(\theta, \lambda)=3 n \log (\theta)-n \log (2+\theta)+\sum_{i}^{n} \log \left[x_{i}\left(x_{i}+1\right)\right]-n \theta \bar{x}+\sum_{i}^{n} \log \left(1-\lambda+2 \lambda\left(\frac{\theta^{2} x_{i}^{2}}{\theta+2}+\theta x+1\right) e^{-\theta x}\right) .
$$

To get the maximum likelihood estimator of $\lambda$ and $\theta$, we have to solve $\frac{\partial L(\theta, \lambda)}{\partial \theta}=0$ and $\frac{\partial L(\theta, \lambda)}{\partial \lambda}=0$ Hence, we have

$$
\frac{3 n}{\theta}-\frac{n}{2+\theta}-n \bar{x}+2 \lambda \sum_{i=1}^{n} \frac{\frac{\left(x_{i}+2 \theta(\theta+2) x_{i}+\theta^{2} x_{i}\right) e^{-\theta x_{i}}}{(\theta+2)^{2}}-x_{i}\left(\frac{\theta^{2} x_{i}^{2}}{\theta+2}+\theta x_{i}+1\right) e^{-\theta x_{i}}}{\left(1-\lambda+2 \lambda\left(\frac{\theta^{2} x_{i}^{2}}{2+\theta}+\theta x_{i}+1\right) e^{-\theta x_{i}}\right)}=0
$$


and

$$
\sum_{i=1}^{n}\left(\frac{2\left(\frac{\theta^{2} x_{i}^{2}}{2+\theta}+\theta x_{i}+1\right) e^{-\theta x_{i}}-1}{1-\lambda+2 \lambda\left(\frac{\theta^{2} x_{i}^{2}}{\theta+2}+\theta x_{i}+1\right) e^{-\theta x_{i}}}\right)=0
$$

To get the MLE of $\lambda$ and $\theta$, we use the R- statistical package to solve these equation numerically.

\section{Simulation Study}

To generate the random sample from transmuted zeghdoudi distribution, we proceed as follows:

Let $F(x)=p$, where $p$ follows uniform $(0,1)$.

i.e.

i.e.

$$
(1+\lambda) G(x)-\lambda G^{2}(x)=p
$$

$$
U=G(x)=\frac{(1+\lambda) \pm \sqrt{\left(1+\lambda^{2}-4 \lambda p\right)}}{2 \lambda},|\lambda| \leq 1
$$

We will choose that solution which lies in $(0,1)$. Here $G(x)$ is the cdf of zeghdoudi random variable. Then, we follow the approach of generating sample from zeghdoudi distribution. Hence the algorithm is

- Generate $p_{i} \sim$ uniform $(0,1), i=1,2, \ldots, n$.

- Calculate $U_{i}=\frac{(1+\lambda) \pm \sqrt{\left(1+\lambda^{2}-4 \lambda p_{i}\right)}}{2 \lambda}, i=1,2, \ldots, n$.

- Generate $V_{i} \sim \operatorname{gamma}(2, \theta), i=1,2, \ldots, n$.

- Generate $W_{i} \sim \operatorname{gamma}(3, \theta), i=1,2, \ldots, n$.

- If $U_{i} \leq \frac{\theta}{2+\theta}$, then set $X_{i}=V_{i}$. Otherwise, set $X_{i}=W_{i}$.

A Monte Carlo simulation study is carried out considering $N=10000$ times for different values of $n$ and presented graphically for $\theta=0.8$ and $\lambda=0.6$ (Figure ). The following two measures were computed

- Average bias of the simulated estimates $\hat{\theta}_{i}, i=1,2, \ldots, N: \frac{1}{N} \sum_{i=1}^{N}\left(\hat{\theta}_{i}-\theta\right)$

- Average Mean Square Error (MSE) of the simulated estimates $\hat{\theta}_{i}, i=1,2, \ldots, N: \frac{1}{N} \sum_{i=1}^{N}\left(\hat{\theta}_{i}-\theta\right)^{2}$

\section{Data Analysis}

In this section,we use a real lifetime data and shown that the flexibility of Transmuted Zeghdoudi Distribution.

Data set 1- represent the waiting times (in mins) before customer service in bank $0.80 .81 .31 .5,1.8,1.9,1.9,2.1,2.6,2.7$, 2.9, 3.1, 3.2, 3.3, 3.5, 3.6, 4, 4.1, 4.2, 4.2, 4.3, 4.3, 4.4, 4.4, 4.6, 4.7, 4.7, 4.8, 4.9, 4.9, 5, 5.3, 5.5, 5.7, 5.7, 6.1, 6.2, 6.2, 6.2, 6.3, 6.7, 6.9, 7.1, 7.1, 7.1, 7.1, 7.4, 7.6, 7.7, 8, 8.2, 8.6, 8.6, 8.6, 8.8, 8.8, 8.9, 8.9, 9.5, 9.6, 9.7, 9.8, 10.7, 10.9, 11, 11, 11.1, $11.2,11.2,11.5,11.9,12.4,12.5,12.9,13,13.1,13.3,13.6,13.7,13.9,14.1,15.4,15.4,17.3,17.3,18.1,18.2,18.4,18.9$, $19,19.9,20.6,21.3,21.4,21.9,23,27,31.6,33.1,38.5$

Table 1: The MLEs of parameter(s), negative log-likelihood and AIC values for two survival model.

\begin{tabular}{|c|c|c|c|}
\hline Survival model & MLE & negative log-likelihood & $\begin{array}{c}\text { AIC } \\
\text {-2log-likelihood }+2 \mathrm{~K} \\
\text {,K=parameter }\end{array}$ \\
\hline $\begin{array}{c}\text { Transmuted } \\
\text { Zeghdoudi }(\boldsymbol{\theta}, \boldsymbol{\lambda})\end{array}$ & $\begin{array}{c}\hat{\theta}=0.2593, \\
\hat{\lambda}=0.3784\end{array}$ & 314.271 & 632.542 \\
\hline Zeghdoudi $(\boldsymbol{\theta})$ & $\hat{\theta}=0.3886$ & 334.234 & 670.468 \\
\hline Lindley $(\boldsymbol{\theta})$ & $\hat{\theta}=0.1865$ & 319.0468 & 640.093 \\
\hline Exponential $(\boldsymbol{\theta})$ & $\hat{\theta}=0.1012$ & 329.047 & 660.094 \\
\hline Xgamma $(\boldsymbol{\theta})$ & $\hat{\theta}=0.2633$ & 332.237 & 666.474 \\
\hline
\end{tabular}


The curve fitting to waiting time

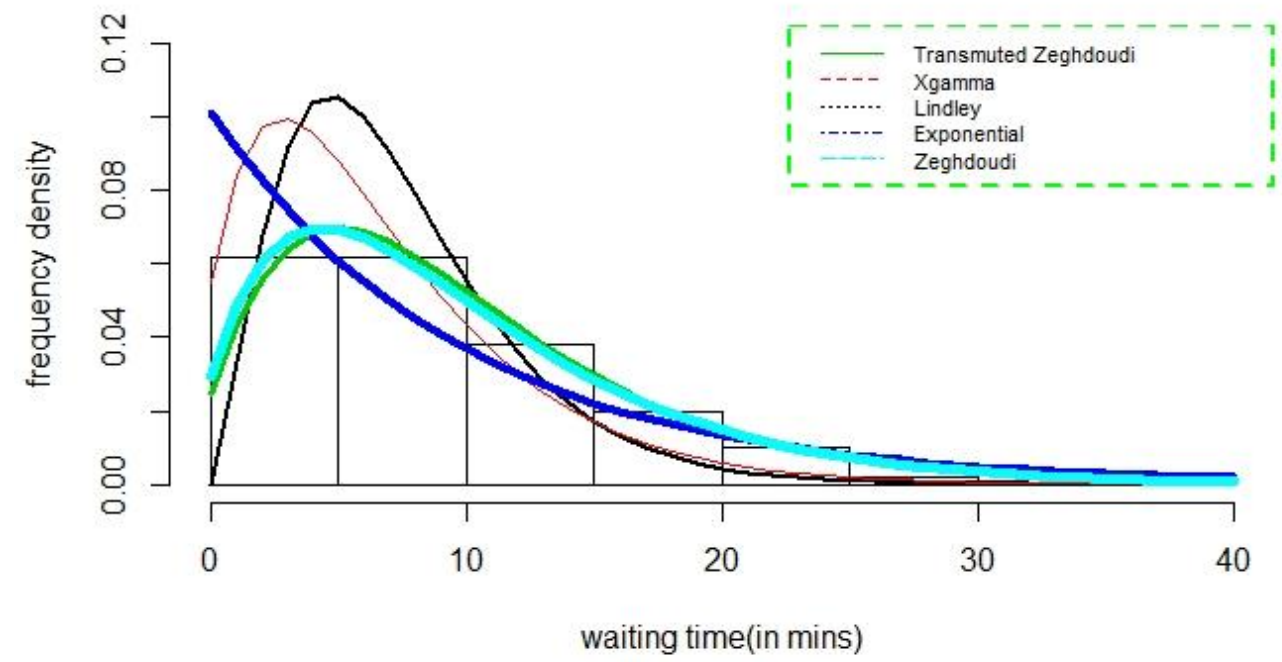

Figure 5: The graph showing fitting of the different distribution to the waiting time(in mins) before the customer service.

\section{Conclusion}

In this article, we introduce a new two parameter Transmuted Zeghdoudi Distribution(TZD) which is an extension of one parameter Zeghdoudi Distribution. We derived some statistical properties, estimation of parameters and application to some real lifetime data sets. An application of the proposed distribution to the "waiting times before customer service in bank" data are given to show that the new distribution provides better fit than other models available in the paper. We expect that this article will serve as a reference for many researchers for future research in this field.

\section{Aknowledgements}

The author is grateful to Prof. Sudhansu Sekhar Maiti, Visva Bharati University who helped him a lot and also thakful to the unknown referee and Editor-In Chief for their helpful and valuable comments and suggestions for publishing the paper.

\section{References}

[1] Alfred Akinsete, Felix Famoye, and Carl Lee. “The beta-pareto distribution”. Statistics, 42(6):547-563, 2008.

[2] Mohamed E Ghitany, Barbra Atieh, and Saralees Nadarajah. "Lindley distribution and its application". Mathematics and computers in simulation, 78(4):493-506, 2008.

[3] Tomasz J Kozubowski and Saralees Nadarajah. "The beta-laplace distribution”. Journal of Computational Analysis \& Applications, 10(1), 2008.

[4] Subhradev Sen, Sudhansu S Maiti, and N Chandra. "The xgamma distribution: statistical properties and application". Journal of Modern Applied Statistical Methods, 15(1):38, 2016.

[5] Muhammad H Tahir, Gauss M Cordeiro, Ayman Alzaatreh, Muhammad Mansoor, and Muhammad Zubair. "A new weibull-pareto distribution: properties and applications". Communications in Statistics-Simulation and Computation, 45(10):3548-3567, 2016.

[6] Luz M Zea, Rodrigo B Silva, Marcelo Bourguignon, Andrea M Santos, and Gauss M Cordeiro. "The beta exponentiated pareto distribution with application to bladder cancer susceptibility". International Journal of Statistics and Probability, 1(2):8, 2012.

[7] CC Odom, EC Nduka, and MA Ijomah. "A modification of pranav distribution using quadratic rank transmutation map approach". Int. J. Sci. Res. in Mathematical and Statistical Sciences Vol, 6:2, 2019.

[8] Faton Merovci. "Transmuted lindley distribution”. International Journal of Open Problems in Computer Science and Mathematics, 238(1393):1-20, 2013.

[9] Halim Zeghdoudi and Sihem Nedjar. "Gamma lindley distribution and its application”. Journal of Applied Probability and Statistics, 11(1):129-138, 2016. 
[10] Hamouda Messaadia and Halim Zeghdoudi. “Zeghdoudi distribution and its applications”. International Journal of Computing Science and Mathematics, 9(1):58-65, 2018.

[11] William T Shaw and Ian RC Buckley. "The alchemy of probability distributions: beyond gram-charlier expansions, and a skew-kurtoticnormal distribution from a rank transmutation map". arXiv preprint arXiv:0901.0434, 2009.

[12] AP Prudnikov, Yu A Brychkov, and OI Marichev. “Integrals and series” [in russian], vol. 1. Elementary Functions: Complementary Chapters, $1,1981$.

[13]Gagan Gurung, Rahul Shah, Dhiraj Prasad Jaiswal, "Emerging Trends in Data Mining- An Algorithms used, Challenges and its Significance in Current Scenario," International Journal of Computer Sciences and Engineering, Vol.8, Issue.3, pp.78-82, 2020.

[14]Logunleko K.B., Logunleko A.M., Odufowora M.O., Gbolagade, K.A., "A Differential Computational Encryption Modeling Technique on Textual Data," International Journal of Scientific Research in Computer Science and Engineering, Vol.8, Issue.1, pp.81-86, 2020

\section{AUTHORS PROFILE}

Molay Kumar Ruidas completed B.Sc. \& M. Sc. Statistics from Visva Bharati University, Shantiniketan in 2015 \& 2017 respectively. He is currently working as an Assistant Professor in Department of Statistics, Trivenidevi Bhalotia College, Raniganj since 2019. Also he is the Ph.D Research scholar of West Bengal State University, Barasat. His main research work focuses on Probability Distribution, Statistical Inference based on his education.

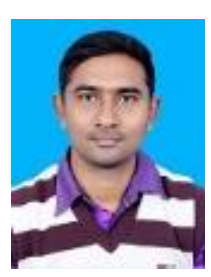

